Research Article

\title{
Some Properties of Bifractional Bessel Processes Driven by Bifractional Brownian Motion
}

\author{
Xichao Sun $\mathbb{D}^{1},{ }^{1}$ Rui Guo $\mathbb{D}^{2}{ }^{2}$ and Ming Li $\mathbb{D}^{3,4}$ \\ ${ }^{1}$ Department of Mathematics, College of Science, Bengbu University, 1866 Caoshan Rd., Bengbu 233030, China \\ ${ }^{2}$ College of Information Science and Technology, Donghua University, 2999 North Renmin Rd., Songjiang, \\ Shanghai 201620, China \\ ${ }^{3}$ Ocean College, Zhejiang University, Zhejiang 310012, China \\ ${ }^{4}$ Shanghai Key Laboratory of Multidimensional Information Processing, East China Normal University, \\ No. 500 Dong-Chuan Road, Zhongshan, Shanghai 200241, China
}

Correspondence should be addressed to Ming Li; ming_lihk@yahoo.com

Received 1 May 2020; Accepted 24 September 2020; Published 19 October 2020

Academic Editor: Wenguang Yu

Copyright (c) 2020 Xichao Sun et al. This is an open access article distributed under the Creative Commons Attribution License, which permits unrestricted use, distribution, and reproduction in any medium, provided the original work is properly cited.

Let $\left(B=\left\{\left(B_{t}^{1}, \ldots, B_{t}^{d}\right)\right\}_{t \geq 0}\right)$ be a $d$-dimensional bifractional Brownian motion and $R_{t}=\sqrt{\left(B_{t}^{1}\right)^{2}+\cdots+\left(B_{t}^{d}\right)^{2}}$ be the bifractional Bessel process with the index $(2 \mathrm{HK} \geq 1)$. The Itô formula for the bifractional Brownian motion leads to the equation $\left(R_{t}=\sum_{i=1}^{d} \int_{0}^{t}\left(B_{s}^{i} / R_{s}\right) \mathrm{d} B_{s}^{i}+\mathrm{HK}(d-1) \int_{0}^{t}\left(s^{2 \mathrm{HK}-1} / R_{s}\right) \mathrm{d} s\right)$. In the Brownian motion case $(K=1) \quad$ and $\quad(H=(1 / 2))$, $\left(X_{t}:=\sum_{i=1}^{d} \int_{0}^{t}\left(B_{s}^{i} / R_{s}\right) \mathrm{d} B_{s}^{i}, \quad d \geq 1\right)$ is a Brownian motion by Lévy's characterization theorem. In this paper, we prove that process $X_{t}$ is not a bifractional Brownian motion unless $(K=1)$ and $(H=(1 / 2))$. We also study some other properties and their application of this stochastic process.

\section{Introduction}

Given $H \in(0,1)$ and $K \in[0,1]$, the bifractional Brownian motion with the indices $H$ and $K$ is a mean zero Gaussian process $B=\left\{B_{t}^{H, K}, t \geq 0\right\}$ such that $B_{0}^{H, K}=0$ and

$$
E\left[B_{s}^{H, K} B_{t}^{H, K}\right]=R(t, s):=\frac{1}{2^{K}}\left[\left(t^{2 H}+s^{2 H}\right)^{K}-|t-s|^{2 \mathrm{HK}}\right],
$$

for all $(s, t \geq 0)$. This process was first introduced by Houdré and Villa [1]. More works for bifractional Brownian motion and their application can be found in [2-10] and the references therein. Clearly, the process is a fractional Brownian motion with Hurst the parameter $H$ when $K=1$. Particularly, the process is a Brownian motion when $(K=1)$ and $(H=(1 / 2))$. Since $\left(B_{t}^{H, K}\right)$ is neither a Markov process nor a semimartingale unless $(K=1)$ and $(H=(1 / 2))$, a lot of powerful techniques from classical stochastic analysis are not available to deal with it. As the generalization of the fractional Brownian motion, the bifractional Brownian motion also admits Hölder paths and selfsimilarity, but its increments are not stationary.

Let $B=\left(B^{1}, \ldots, B^{d}\right)$ be a $d$-dimensional bifractional Brownian motion with the index $(H K \geq(1 / 2))$. That is to say, each component of $B$ is an independent one-dimensional bifractional Brownian motion with the index $(\mathrm{HK} \geq(1 / 2))$. Let $R_{t}$ be the bifractional Bessel process defined by $R_{t}=\sqrt{\left(B_{t}^{1}\right)^{2}+\cdots+\left(B_{t}^{d}\right)^{2}}$.

There is an extensive literature on this process for the standard Brownian motion case $(K=1$ and $H=(1 / 2))$ and the fractional Brownian motion case $(K=1)$ (see [11-15]). For $(d \geq 2, \mathrm{HK}>(1 / 2))$, by the Itô formula for the bifractional Brownian motion, we have (see Alós et al. [16] and EsSebaiy and Tudor [3])

$$
R_{t}=\sum_{i=1}^{d} \int_{0}^{t} \frac{B_{s}^{i}}{R_{s}} \mathrm{~d} B_{s}^{i}+\mathrm{HK}(d-1) \int_{0}^{t} \frac{s^{2 \mathrm{HK}-1}}{R_{s}} \mathrm{~d} s
$$

and for $d=1$ and $(\mathrm{HK} \geq(1 / 2))$, one also has 


$$
\left|B_{t}^{H, K}\right|=\int_{0}^{t} \operatorname{sign}\left(B_{s}^{H, K}\right) \mathrm{d} B_{s}^{H, K}+\mathrm{HK} \int_{0}^{t} \delta\left(B_{s}^{H, K}\right) s^{2 \mathrm{HK}-1} \mathrm{~d} s
$$

where stochastic integrals are interpreted in the divergence sense and $\delta$ denotes the Dirac delta function. When $K=1$ and $H=(1 / 2)$, the process

$$
X_{t}= \begin{cases}\int_{0}^{t} \operatorname{sign}\left(B_{s}^{H, K}\right) \mathrm{d} B_{s}^{H, K}, & d=1, \\ \sum_{i=1}^{d} \int_{0}^{t} \frac{B_{s}^{i}}{R_{s}} \mathrm{~d} B_{s}^{i}, & d \geq 2\end{cases}
$$

is a standard Brownian motion by Lévy's characterization theorem. Given $K=1$, the fractional Brownian motion case was researched by $\mathrm{Hu}$ and Nualart [11]. So, it is natural and interesting to research the process $X=\left\{X_{t}, t \geq 0\right\}$ for more general $H$ and $K$. Since there is no characterization as convenient as Lévy's characterization theorem for general bifractional Brownian motion, to prove a stochastic process is a bifractional Brownian motion or not is difficult. The method used here is essentially based on $\mathrm{Hu}$ and Nualart [11] and Shen et al. [17]. It is not difficult to find that the bifractional Brownian motion has the nonavailability of convenient stochastic integral representations and more complexity of dependence structures than an fractional Brownian motion and a subfractional Brownian motion. Therefore, it seems interesting to study bifractional Bessel processes driven by bifractional Brownian motions.

The rest of the paper is organized as follows. In Section 2, we present some preliminaries for the bifractional Brownian motion. In Section 3, some properties to the process $\int_{0}^{t} \operatorname{sign}\left(B_{t}^{H, K}\right) \mathrm{d} B_{t}^{H, K}$ are studied. In Section 4 , we consider the process $\left(\sum_{i=1}^{d} \int_{0}^{t}\left(B_{s}^{i} / R_{s}\right) \mathrm{d} B_{s}^{i}\right)$ with $d \geq 2$. In Section 5 , we consider the local time and Tanaka formula of the process $\int_{0}^{t} \operatorname{sign}\left(B_{t}^{H, K}\right) \mathrm{d} B_{t}^{H, K}$.

\section{Preliminaries}

In this paper, we assume that $((1 / 2)<\mathrm{HK}<1)$ is arbitrary but fixed and let $B=\left\{B_{t}^{H, k}, \quad 0 \leq t \leq T\right\}$ be a bifractional Brownian motion with the index $H$ and $K$, which is defined on the complete probability space $(\Omega, \mathscr{F}$, and $P)$. One can construct a stochastic calculus of variations with respect to the bifractional Brownian motion $B^{H, k}$ by the Malliavin calculus method (see Alòs et al. [16] and Nualart [18]). We next recall the basic definitions and results for this calculus.

Bifractional Brownian motion $B^{H, k}$ satisfies the estimates:

$$
2^{-K}|t-s|^{2 \mathrm{HK}} \leq E\left[\left(B_{t}-B_{s}\right)^{2}\right] \leq 2^{1-K}|t-s|^{2 \mathrm{HK}} .
$$

One can write its covariance as follows:

$$
R(t, s)=R_{1}(t, s)+R_{2}(t, s),
$$

where

$$
\begin{aligned}
& R_{1}(t, s)=\frac{1}{2^{K}}\left[\left(s^{2 H}+t^{2 H}\right)^{K}-\left(s^{2 \mathrm{HK}}+t^{2 \mathrm{HK}}\right)\right], \\
& R_{2}(t, s)=\frac{1}{2^{K}}\left[t^{2 \mathrm{HK}}+s^{2 \mathrm{HK}}-|t-s|^{2 \mathrm{HK}}\right] .
\end{aligned}
$$

Therefore,

$$
\begin{aligned}
& \frac{\partial^{2}}{\partial t \partial s} R_{1}(t, s)=4 H^{2} K(K-1) 2^{-K} t^{2 H-1} s^{2 H-1}\left(t^{2 H}+s^{2 H}\right)^{K-2} \\
& \frac{\partial^{2}}{\partial t \partial s} R_{2}(t, s)=2(2 \mathrm{HK}-1) \mathrm{HK}|t-s|^{2 \mathrm{HK}-2} .
\end{aligned}
$$

Since $R_{1}$ is of the class $C^{2}\left([0, T]^{2}\right)$ and $\left(\partial^{2} / \partial t \partial s\right) R_{1}(t, s)$ is always negative, $R_{1}$ is the distribution function and has $\left(\partial^{2} / \partial t \partial s\right) R_{1}(t, s)$ for density. $R_{2}$ is the distribution function with density $\left(\partial^{2} / \partial r \partial s\right) R_{1}(t, s)=2(2 \mathrm{HK}-1) \mathrm{HK}|t-s|^{2 \mathrm{HK}-2}$ and belongs to $L^{1}\left([0, T]^{2}\right)$. It follows that there exist two positive constants $c_{H, K}$ and $C_{H, K}$ which satisfy

$$
c_{H, K}|t-s|^{2 \mathrm{HK}-2} \leq\left|\frac{\partial^{2}}{\partial t \partial s} R(t, s)\right| \leq C_{H, K}|t-s|^{2 \mathrm{HK}-2} .
$$

\section{Denote}

$$
\phi(t, s)=(2 \mathrm{HK}-1) \mathrm{HK}|t-s|^{2 \mathrm{HK}-2}, \quad \text { for } s, t \geq 0 .
$$

As a Gaussian process of $B^{H, k}$, we can construct a stochastic calculus of variations with respect to this process. Suppose that $\mathscr{H}$ is the completion of the space $\mathscr{E}$ which is generated by $\left\{1_{[0, T]}, t \in[0, T]\right\}$ with respect to the following inner product:

$$
\left\langle 1_{[0, s]}, 1_{[0, t]}\right\rangle_{\mathscr{H}}=R(s, t) .
$$

Then, $\varphi \in \mathscr{E} \mapsto B(\varphi)$ is an isometry from $\mathscr{E}$ to the Gaussian space generated by $B$ which can be extended to $\mathscr{H}$. We can write this Hilbert space $\mathscr{H}$ as follows:

$$
\mathscr{H}=\left\{\varphi:[0, T] \longrightarrow \mathbb{R} \mid\|\varphi\|_{\mathscr{H}}<\infty\right\}
$$

where $\|\varphi\|_{\mathscr{H}}^{2}:=\int_{0}^{T} \varphi(s) \varphi(r) \phi(s, r) \mathrm{d} s \mathrm{~d} r$. We can define the spaces of measurable functions as follows:

$$
|\mathscr{H}|=\left\{\varphi:[0, T] \longrightarrow \mathbb{R} \mid\|\varphi\|_{|\mathscr{H}|}<\infty\right\},
$$

where

$$
\|\varphi\|_{|\mathscr{H}|}^{2}:=\int_{0}^{T} \int_{0}^{T}|\varphi(s) \| \varphi(r)| \phi(s, r) \mathrm{d} s \mathrm{~d} r<\infty
$$

It is easy to see that $\mathscr{E}$ is dense in $|\mathscr{H}|$ and $|\mathscr{H}|$ is a Banach space. Suppose that $\mathcal{S}$ is the set of smooth functional

$$
F=f\left(B^{H, K}\left(\varphi_{1}\right), B^{H, K}\left(\varphi_{2}\right), \ldots, B^{H, K}\left(\varphi_{2}\right)\right),
$$

where $f \in C_{b}^{\infty}\left(\mathbb{R}^{n}\right)$ and $\varphi_{i} \in \mathscr{H}$. The Malliavin derivative $D$ of the above functional $F$ is given as follows: 


$$
\mathrm{DF}=\sum_{j=1}^{n} \frac{\partial f}{\partial x_{j}}\left(B^{H, K}\left(\varphi_{1}\right), B^{H, K}\left(\varphi_{2}\right), \ldots, B^{H, K}\left(\varphi_{n}\right)\right) \varphi_{j} .
$$

The derivative operator $D$ is a closable operator from space $L^{2}(\Omega)$ into space $L^{2}(\Omega ; \mathscr{H})$. We denote $\mathbb{D}^{1,2}$, the closure of $\mathcal{S}$, with respect to norm

$$
\|F\|_{1,2}:=\sqrt{E|F|^{2}+E\|\mathrm{DF}\|_{\mathscr{H}}^{2}} .
$$

The divergence integral $\delta$ is the adjoint operator of $D$. $\delta(u)$ can be defined by the duality relationship:

$$
E[F \delta(u)]=E\langle D F, u\rangle_{\mathscr{H}},
$$

for any $u \in \mathbb{D}^{1,2}$. For any $u \in \mathbb{D}^{1,2}$, one has $\left(\mathbb{D}^{1,2} \subset \operatorname{Dom}(\delta)\right)$ and

$$
\begin{aligned}
E\left[\delta(u)^{2}\right] & =E\|u\|_{\mathscr{H}}^{2}+E \int_{[0, T]^{4}} D_{\xi} u_{r} D_{\eta} u_{s} \phi(\xi, s) \phi(\eta, r) \mathrm{d} s \mathrm{~d} r \mathrm{~d} \xi \mathrm{d} \eta \\
& \leq E\|u\|_{|\mathscr{H}|}^{2}+E \int_{[0, T]^{4}}\left|D_{\xi} u_{r} \| D_{\eta} u_{s}\right| \phi(\xi, s) \phi(\eta, r) \mathrm{d} s \mathrm{~d} r \mathrm{~d} \xi \mathrm{d} \eta
\end{aligned}
$$

where

$$
\delta(u)=\int_{0}^{T} u_{s} \mathrm{~d} B_{s}^{H, K}
$$

expressing the Skorokhod integral of a process $u$.

\section{Case of One Dimension}

We study the stochastic process $X=\left\{X_{t}, \quad t \geq 0\right\}$ defined by

$$
X_{t}=\int_{0}^{t} \operatorname{sign}\left(B_{s}^{H, K}\right) \mathrm{d} B_{s}^{H, K} .
$$

If $K=1$ and $H=(1 / 2), X_{t}$ is a standard Brownian motion from Levy's characterization theorem. It is then natural to study any parameter $H$ and $K$. Next, we first prove $X$ is an HK-self-similar process for any $H K \geq(1 / 2)$.

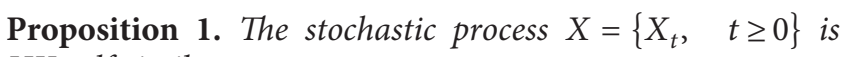
HK-self-similar.

Proof. Together with the HK-self-similarity property of the bifractional Brownian motion and Tanaka formula (4), for any $a>0$, one can obtain

$$
\begin{aligned}
X_{\mathrm{at}} & =\left|B_{\mathrm{at}}^{H, K}\right|-\mathrm{HK} \int_{0}^{a t} \delta\left(B_{s}^{H, K}\right) B_{s}^{2 \mathrm{HK}-1} \mathrm{~d} s \\
& =\left|B_{\mathrm{at}}^{H, K}\right|-\mathrm{HK} \int_{0}^{t} \delta\left(B_{\mathrm{au}}^{H, K}\right)(\mathrm{au})^{2 \mathrm{HK}-1} a \mathrm{~d} u \\
& \stackrel{d}{=} a^{\mathrm{HK}}\left|B_{\mathrm{t}}^{H, K}\right|-\mathrm{HK} a^{2 \mathrm{HK}} \int_{0}^{t} \delta\left(a^{\mathrm{HK}} B_{u}^{H, K}\right)(u)^{2 \mathrm{HK}-1} \mathrm{~d} u \\
& =a^{\mathrm{HK}} X_{t},
\end{aligned}
$$

where $\stackrel{d}{=}$ denotes that both stochastic processes have the same distributions. This proof is completed.

For stochastic process $\operatorname{sign}\left(B_{t}^{H, K}\right)$, we first obtain the Wiener chaos expansion. Let $I_{n}$ be the multiple Wiener integral of the stochastic process $B^{H, K}$.

Proposition 2. For any $t \geq 0$, one can obtain

$$
\operatorname{sign}\left(B_{t}^{H, K}\right)=\sum_{m=0}^{\infty} b_{2 m+1} I_{2 m+1}(1)
$$

where

$$
b_{2 m+1}=\frac{2(-1)^{m}}{(2 m+1) \sqrt{2 \pi} t^{(2 m+1) \mathrm{HK}} m ! 2^{m}}
$$

Proof. For $\varepsilon>0$, we denote

$$
\begin{aligned}
& p_{\varepsilon}(y)=\frac{1}{\sqrt{2 \pi \varepsilon}} e^{-\left(y^{2} / 2 \varepsilon\right)} \\
& f_{\varepsilon}(y)=2 \int_{-\infty}^{y} p_{\varepsilon}(z) \mathrm{d} z-1, \quad y \in \mathbb{R} .
\end{aligned}
$$

Then,

$$
p_{t^{2 \mathrm{HK}}}(y)=\frac{1}{\sqrt{2 \pi} t^{\mathrm{HK}}} \exp \left(-\frac{y^{2}}{2 t^{2 \mathrm{HK}}}\right), \quad x \in \mathbb{R},
$$

which is a density function of the bifractional Brownian motion $B_{t}^{H, K}$ and $f_{\varepsilon}\left(B_{t}^{H, K}\right) \longrightarrow \operatorname{sign}\left(B_{t}^{H, K}\right)$ in $L^{2}(\Omega)$ as $\varepsilon \longrightarrow 0$. By Stroock's formula, one can obtain

$$
f_{\varepsilon}\left(B_{t}^{H, K}\right)=\sum_{m=0}^{\infty} a_{m}^{\varepsilon}(t) \int_{0<s_{1}<\cdots<s_{n}<t} \mathrm{~d} B_{s_{1}}^{H, K}, \ldots, \mathrm{d} B_{s_{n}}^{H, K}
$$

where

$$
\begin{aligned}
a_{m}^{\varepsilon}(t) & =E\left[D^{m}\left(f_{\varepsilon}\left(B_{t}^{H, K}\right)\right)\right]=2 E\left[p_{\varepsilon}^{(m-1)}\left(B_{t}^{H, K}\right)\right] \\
& =\left.2(-1)^{m-1} \frac{\partial^{m-1}}{\partial z^{m-1}} E\left[p_{\varepsilon}\left(B_{t}^{H, K}-z\right)\right]\right|_{z=0} \\
& =2(-1)^{m-1} p_{\varepsilon+t^{2 H K}}^{(m-1)}(0) .
\end{aligned}
$$


As $\varepsilon \longrightarrow 0$, by taking the limit of $(27)$ in the space $L^{2}(\Omega)$, one can obtain

$$
\operatorname{sign}\left(B_{t}^{H, K}\right)=\sum_{m=0}^{\infty} a_{n}(t) \int_{0<s_{1}<\cdots<s_{n}<t} \mathrm{~d} B_{s_{1}}^{H, K}, \ldots, \mathrm{d} B_{s_{n}}^{H, K},
$$

where $a_{m}(t)=\lim _{\varepsilon \longrightarrow 0} a_{m}^{\varepsilon}(t)=2(-1)^{m-1} p_{t^{\mathrm{HK}}}^{(n-1)}(0)$, which implies

$$
a_{m}(t)= \begin{cases}0, & n=2 k, \\ \frac{2(-1)^{k}(2 k) !}{\sqrt{2 \pi} t^{n H} k ! 2^{k}}, & n=2 k+1 .\end{cases}
$$

The proof is completed.

In this paper, the notation $F \asymp G$ implies that there are two positive constants $c_{1}$ and $c_{2}$ such that

$$
c_{1} G(x) \leq F(x) \leq c_{2} G(x) \text {, }
$$

where $C$ denotes a generic positive constant and $F$ and $G$ have the common domain.

Proposition 3. The random variable sign $\left(B_{t}^{H, K}\right)$ belongs to the Sobolev-Watanabe space $\mathbb{D}^{\alpha, 2}$ for any $t \geq 0$ and $\alpha<(1 / 2)$.

Proof. By Stirling's formula

$$
\lim _{k \rightarrow \infty} \frac{k !}{k^{(k+(1 / 2))} e^{-k}}=\sqrt{2 \pi},
$$

we have

$$
\begin{aligned}
E\left[I_{2 m+1}\left(b_{2 m+1}\right)\right]^{2} & =(2 m+1) !\left\langle b_{2 m+1}, b_{2 k+1}\right\rangle_{\mathscr{H}^{22 m+1}} \\
& =\frac{(2 m+1) ! 4\left(t^{2 \mathrm{HK}}\right)^{2 m+1}}{(2 m+1)^{2} 2 \pi t^{(2 m+1) 2 \mathrm{HK}}\left(m ! 2^{m}\right)^{2}} \\
& =\mathrm{Cm}^{-3 / 2}
\end{aligned}
$$

The proof is completed.

Proposition 4. For any $t \geq 0$, one has

$$
\int_{0}^{t} \operatorname{sign}\left(B_{s}^{H, K}\right) \mathrm{d} B_{s}^{H, K}=\sum_{m=1}^{\infty} c_{m} I_{2 m}\left(h_{2 m}\right),
$$

where $c_{m}=(-1)^{m-1}\left(\sqrt{2 \pi}(2 m-1)(m-1) ! 2^{m-2}\right)^{-1}$ and

$$
h_{2 m}\left(B_{1}, \ldots, B_{2 m}\right)=\left(B_{1} \vee B_{2} \vee \cdots \vee B_{2 m}\right)^{-(2 m-1) \mathrm{HK}} \text {. }
$$

The above proposition is the chaos expansion of $\int_{0}^{t} \operatorname{sign}\left(B_{t}^{H, K}\right) \mathrm{d} B_{t}^{H, K}$ and implies the following result, which can be proved by the method similar to Proposition 3.

Proposition 5. For any $\alpha<(1 / 2)$ and $t \geq 0$, the random variable $\int_{0}^{t} \operatorname{sign}\left(B_{s}^{H, K}\right) d B_{s}^{H, K}$ belongs to the Sobolev-Watanabe space $\mathbb{D}^{\alpha, 2}$. Now, we consider the stochastic process $X$ :

$$
\rho(n):=E\left[\left(X_{a+1}-X_{a}\right)\left(X_{n+1}-X_{n}\right)\right],
$$

where $(0<a \leq n)$.

Definition 1. We say a stochastic process $\left(X_{t}\right)_{t \geq 0}$ is longrange dependent (resp. short-range dependent) if for each $a>0$,

$$
\sum_{n \geq a}|\rho(n)|=\infty, \quad\left(\operatorname{resp} . \sum_{n \geq a}|\rho(n)|<\infty\right) .
$$

Theorem 1. The stochastic process $X$ of (21) is short-range dependent. Before proving this theorem, a lemma given by Yan et al. [9] is stated.

Lemma 1. Let $(0 \leq r<s)$ and $(0<H K<1)$, one defines

$$
\rho_{r, s}^{2}:=s^{2 \mathrm{HK}} r^{2 \mathrm{HK}}-\mu^{2},
$$

where $\mu=E\left(B_{s}^{H, K} B_{r}^{H, K}\right)$. Then, we have

$$
\rho_{r, s}^{2}=(s-r)^{2 \mathrm{HK}} r^{2 \mathrm{HK}} \text {. }
$$

Remark 1. The proof of estimate (39) uses the following two inequalities:

$$
\begin{gathered}
(1+x)^{\alpha} \leq 1+\left(2^{\alpha}-1\right) x^{\alpha}, \quad 0 \leq x, \alpha \leq 1, \\
(u+v-1)^{K} \leq u^{K}+v^{K}-1 \leq(u+v-1)^{K}+(u v)^{K}, \quad(0 \leq K \leq 1),
\end{gathered}
$$

where $0 \leq u, v \leq 1$ and $u+v \geq 1$. It is not difficult to prove inequality (40), which is stronger than the well-known inequality

$$
(1+x)^{\alpha} \leq 1+\alpha x^{\alpha} \leq 1+x^{\alpha}
$$

because $\left(2^{\alpha}-1 \leq \alpha\right)$ for all $(0 \leq \alpha \leq 1)$.

Proof of Theorem 1. For $0<a<k$, one can obtain 


$$
\begin{aligned}
\rho(k)= & E\left[\int_{a}^{a+1} \operatorname{sign}\left(B_{t}^{H, K}\right) \mathrm{d} B_{t}^{H, K} \int_{k}^{k+1} \operatorname{sign}\left(B_{t}^{H, K}\right) \mathrm{d} B_{t}^{H, K}\right] \\
= & \int_{a}^{a+1} \int_{k}^{k+1} \phi(t, s) E\left(\operatorname{sign} B_{s}^{H, K} \operatorname{sign} B_{t}^{H, K}\right) \mathrm{d} s \mathrm{~d} t+\int_{a}^{a+1} \int_{k}^{k+1} \int_{0}^{s} \int_{0}^{t} \phi(s, u) \phi(t, v) E\left[\delta\left(B_{s}^{H, K}\right) \delta\left(B_{t}^{H, K}\right)\right] \mathrm{d} u \mathrm{~d} v \mathrm{~d} s \mathrm{~d} t \\
= & \int_{a}^{a+1} \int_{k}^{k+1} \phi(t, s) E\left(\operatorname{sign} B_{s}^{H, K} \operatorname{sign} B_{t}^{H, K}\right) \mathrm{d} s \mathrm{~d} t+4(\mathrm{HK})^{2} \int_{a}^{a+1} \int_{k}^{k+1} \psi_{1}(s, t) \psi_{2}(s, t) E\left[\delta\left(B_{s}^{H, K}\right) \delta\left(B_{t}^{H, K}\right)\right] \mathrm{d} s \mathrm{~d} t \\
= & \int_{a}^{a+1} \int_{k}^{k+1} \phi(t, s) E\left(\frac{B_{s}^{H, K}}{\left|B_{s}^{H, K}\right|} \frac{B_{t}^{\mathrm{HK}}}{\left|B_{t}^{\mathrm{HK}}\right|}\right) \mathrm{d} \mathrm{d} t+4(\mathrm{HK})^{2} \int_{a}^{a+1} \int_{n}^{n+1} \psi_{1}(s, t) \psi_{2}(s, t) E\left[\delta\left(B_{s}^{H, K}\right) \delta\left(B_{t}^{H, K}\right)\right] \mathrm{d} s \mathrm{~d} t \\
= & \int_{a}^{a+1} \int_{k}^{k+1} E\left(\frac{B_{s}^{H, K}}{\left|B_{s}^{H, K}\right|} \frac{B_{t}^{\mathrm{HK}}}{\left|B_{t}^{\mathrm{HK}}\right|}\right)^{\phi(t, s) \mathrm{d} s \mathrm{~d} t} \\
& +4(\mathrm{HK})^{2} \int_{a}^{a+1} \int_{k}^{k+1} E\left[\delta\left(B_{s}^{H, K}\right) \delta\left(B_{t}^{H, K}\right)\right] \cdot\left[s^{2 \mathrm{HK}-1}+(t-s)^{2 \mathrm{HK}-1}\right]\left[t^{2 \mathrm{HK}-1}+(t-s)^{2 \mathrm{HK}-1}\right] \mathrm{d} s \mathrm{~d} t \\
\equiv & a_{k}+b_{k} .
\end{aligned}
$$

Now, we only need to estimate $a_{k}$ and $b_{k}$. For $a_{k}$, by the orthogonal decomposition,

$$
B_{t}^{H, K} \stackrel{d}{=} \frac{\theta_{H}(s, t)}{\theta_{H}(s, s)} B_{s}^{H, K}+\beta_{s, t} \mathcal{N}
$$

where

$$
\beta_{s, t}^{2}=\frac{\rho_{s, t}^{2}}{s^{2 \mathrm{HK}}}
$$

in which $\mathcal{N} \in N(0,1)$ independent of $B_{s}^{H, K}$. Set

$$
\lambda_{s, t}=\frac{\theta_{H}(s, t)}{\beta_{s, t} s^{2 \mathrm{HK}}}=\frac{1 / 2^{K}\left[\left(t^{2 H}+s^{2 H}\right)^{K}-|t-s|^{2 \mathrm{HK}}\right]}{s^{\mathrm{HK}} \rho_{s, t}} .
$$

Since

$$
\left(1+x^{2 H}\right)^{K}-(1-x)^{2 \mathrm{HK}} \sim x^{2 \mathrm{HK}},
$$

$x \longrightarrow 0$. By Lemma 1 , we obtain

$$
\lambda_{s, t}=\frac{\theta_{H}(s, t)}{\beta_{s, t} s^{2 \mathrm{HK}}} \sim C_{H} t^{-\mathrm{HK}}
$$

as $t \longrightarrow \infty$ and $s \in(0,1)$, which implies

$$
\begin{aligned}
E\left(\frac{B_{s}^{H, K} B_{t}^{H, K}}{\left|B_{s}^{H, K} \| B_{t}^{H, K}\right|}\right) & =E\left(\frac{B_{s}^{H, K}\left(\lambda_{s, t} B_{s}^{H, K}+\mathcal{N}\right)}{\left|B_{1}^{H, K} \| \lambda_{s, t} B_{s}^{H, K}+\mathcal{N}\right|}\right), \\
& =E\left(\frac{B_{1}^{H, K}\left(s^{H, K} \lambda_{s, t} B_{1}^{H, K}+\mathcal{N}\right)}{\left|B_{1}^{H, K} \| s^{H, K} \lambda_{s, t} B_{1}^{H, K}+\mathcal{N}\right|}\right) \\
& =O\left(t^{-\mathrm{HK}}\right) .
\end{aligned}
$$

So, the term $a_{k}$ behaves as $k^{2 \mathrm{HK}-3} o\left(k^{-\mathrm{HK}}\right)$. Now, we evaluate the second term $b_{n}$. For $s<t$, using Lemma 1, one can obtain

$$
\begin{aligned}
E\left[\delta\left(B_{s}^{H, K}\right) \delta\left(B_{t}^{H, K}\right)\right] & =\int_{R^{2}} h(y, z) \delta(y) \delta(z) \mathrm{d} y \mathrm{~d} z=h(0,0) \\
& =\frac{1}{2 \pi \rho_{t, s}} \asymp s^{-\mathrm{HK}}(t-s)^{-\mathrm{HK}},
\end{aligned}
$$

where $h(y, z)$ is the density function of $\left(B_{s}^{H, K}, B_{t}^{H, K}\right)$. So,

$$
\begin{aligned}
b_{k} & =\frac{4(\mathrm{HK})^{2}}{2 \pi} \int_{a}^{a+1} \int_{k}^{k+1} \frac{\left[s^{2 \mathrm{HK}-1}+(t-s)^{2 \mathrm{HK}-1}\right]\left[t^{2 \mathrm{HK}-1}+(t-s)^{2 \mathrm{HK}-1}\right]}{\left[(\mathrm{st})^{\mathrm{HK}}-(1 / 4)\left(\left(1 / 2^{K}\right)\left[\left(t^{2 \mathrm{H}}+s^{2 \mathrm{H}}\right)^{K}-|t-s|^{2 \mathrm{HK}}\right]\right)^{2}\right]^{(1 / 2)}} \mathrm{d} \mathrm{d} t \\
& =\int_{a}^{a+1} \int_{k}^{k+1} \frac{\left[(a+1)^{2 \mathrm{HK}-1}+(k+1)^{2 \mathrm{HK}-1}\right](k-a-1)^{2 \mathrm{HK}-1}}{\left[(\mathrm{ak})^{\mathrm{HK}}-(1 / 4)\left(\left(1 / 2^{K}\right)\left[\left((k+1)^{2 \mathrm{H}}+(a+1)^{2 \mathrm{HK}}\right)^{K}-|k-a|^{2 \mathrm{HK}}\right]\right)^{2}\right]^{(1 / 2)} \mathrm{d} \mathrm{d} t} \\
& =k^{3 \mathrm{HK}-3} .
\end{aligned}
$$

The proof is completed. 


\section{Case of Multidimension}

We now consider the $d$-dimensional bifractional Brownian motion $B=\left\{\left(B_{t}^{1}, \ldots, B_{t}^{d}\right)\right\}_{t \geq 0}$ with the index $H K \geq(1 / 2)$, which implies the components $\left(B^{i}, \quad i=1, \ldots, d\right)$ are independent bifractional Brownian motions with the same index $H K \geq(1 / 2)$. As in Section 2, we can define the derivative and divergence operators, $D^{i}$ and $\delta^{i}$, with respect to each component $B^{i}$. Suppose that $\left(\mathbb{D}_{i}^{1, p}(\mathscr{H})\right)$ are the associated Sobolev spaces. Similarly, $\left(\mathbb{L}_{\mathrm{HK}, i}^{1, p}\right)$ denotes the set of processes $u$ in $\left(\mathbb{D}_{i}^{1, p}(|\mathscr{H}|)\right)$ which satisfies

$$
|u|_{\mathbb{L}_{\mathrm{HK}, i}^{1, p}}^{p}:=E\left[|u|_{L^{(1 / \mathrm{HK})}([0, T])}^{p}\right]+E\left[\left\|D^{i} u\right\|_{L^{(1 / \mathrm{HK})}\left([0, T]^{2}\right)}^{p}\right]<\infty .
$$

Let

$$
R_{t}=\left|B_{t}\right|=\sqrt{\left(B_{t}^{1}\right)^{2}+\cdots+\left(B_{t}^{d}\right)^{2}}
$$

be a bifractional Bessel process. In the following, we research the stochastic process:

$$
X_{t}=\sum_{i=1}^{d} \int_{0}^{t} \frac{B_{s}^{i}}{R_{s}} \mathrm{~d} B_{s}^{i}
$$

The next theorem can be proved similar to Es-Sebaiy and Tudor [3].

Theorem 2. Let $B_{t}=\left(B_{t}^{1}, B_{t}^{2}, \ldots, B_{t}^{d}\right)$ be a d-dimensional bifractional Brownian motion with $(2 H K>1)$ and $f$ be a function of class $C^{2}\left(\mathbb{R}^{d}\right)$. Then,

$$
f\left(B_{t}\right)=f(0)+\sum_{i=1}^{d} \int_{0}^{t} \frac{\partial f}{\partial x_{i}}\left(B_{s}\right) \mathrm{d} B_{s}^{i}+\mathrm{HK} \sum_{i=1}^{d} \int_{0}^{t} \frac{\partial^{2} f}{\partial x_{i}^{2}}\left(B_{s}\right) s^{2 \mathrm{HK}-1} \mathrm{~d} s .
$$

The following proposition gives an integral representation for bifractional Bessel processes and can be proved along the lines of the proof of Proposition 5.2. in Guerra and Nualart [19].

Proposition 6. Suppose that $R=\left\{R_{t}\right\}_{t \geq 0}$ is a bifractional Bessel process associated to the $d$-dimensional bifractional Brownian motion with index $H K>(1 / 2)$. For each $(i=1, \ldots, d)$, one can obtain $\left\{\left(B_{s}^{i} / R_{s}\right)\right\}_{s \in[0, T]} \in \mathbb{L}_{H, i}^{(1,1 / H K)}$ and

$$
R_{t}=\sum_{i=1}^{d} \int_{0}^{t} \frac{B_{s}^{i}}{R_{s}} \mathrm{~d} B_{s}^{i}+\mathrm{HK}(d-1) \int_{0}^{t} \frac{B_{s}^{2 \mathrm{HK}-1}}{R_{s}} \mathrm{~d} s .
$$

Proof

Step1. We prove $\int_{0}^{t}\left(B_{s}^{i} / R_{s}\right) \mathrm{d} B_{s}^{i}$ are well defined which only proves $\left\{\left(B_{s}^{i} / R_{s}\right)\right\}_{s \in[0, T]} \in \mathbb{L}_{\mathrm{HK}, i}^{(1,1 / \mathrm{HK})}$ for each $(i=1, \ldots, d)$. Since $\left|B_{s}^{i} / R_{s}\right| \leq 1$ for each $(i=1, \ldots, d)$, one can obtain

$$
E \int_{0}^{T}\left|\frac{B_{s}^{i}}{R_{s}}\right|^{(1 / \mathrm{HK})} \mathrm{d} s<\infty, \quad i=1, \ldots, d .
$$

Together with the definition of the derivative operator and the self-similarity of the bifractional Brownian motion, one can obtain

$$
\begin{aligned}
E \int_{0}^{T} \int_{0}^{T}\left|D_{s}^{i}\left(\frac{B_{r}^{i}}{R_{r}}\right)\right|^{(1 / \mathrm{HK})} \mathrm{d} s \mathrm{~d} r & =\int_{0}^{T} r E\left|\frac{1}{R_{r}}-\frac{\left(B_{r}^{i}\right)^{2}}{R_{r}^{3}}\right|^{(1 / \mathrm{HK})} \mathrm{d} r \\
& \leq \int_{0}^{T} r E\left[R_{r}^{-(1 / \mathrm{HK})}\right] \mathrm{d} r=\mathrm{TE}\left[R_{1}^{-(1 / \mathrm{HK})}\right]=C \int_{0}^{\infty} \frac{1}{(2 \pi)^{(d / 2)}} e^{-\left(u^{2} / 2\right)} u^{d-1-(1 / \mathrm{HK})} \mathrm{d} u<\infty
\end{aligned}
$$

since $(d-1-(1 / \mathrm{HK})>-1)$. So, the integral $\int_{0}^{t}\left(B_{s}^{i} / R_{s}\right) \mathrm{d} B_{s}^{i}$ is well defined since $\left\{\left(B_{s}^{i} / R_{s}\right)\right\}_{s \in[0, T]} \in \mathbb{L}_{H, K, i}^{(1,1 / \mathrm{HK})}$ for each $(i=1, \ldots, d)$.

Step 2. We now prove (56). Note that $f: \mathbb{R}^{d} \longrightarrow \mathbb{R}$ is defined by

$$
f(x)=\sqrt{x_{1}^{2}+\cdots+x_{d}^{2}}
$$

which is not differentiable at the origin. So, we cannot apply the Itô formula (55) to $f$. But, if one considers the square of the bifractional Bessel process

$$
R_{t}^{2}=\left(B_{t}^{1}\right)^{2}+\cdots+\left(B_{t}^{d}\right)^{2}
$$

then one can apply the Itô formula (55), and we have

$$
R_{t}^{2}=2 \sum_{i=1}^{d} \int_{0}^{t} B_{s}^{i} \mathrm{~d} B_{s}^{i}+\mathrm{HKd} t^{2 \mathrm{HK}}
$$

Set

$$
g_{\varepsilon}(y)= \begin{cases}\frac{3}{8} \sqrt{\varepsilon}+\frac{3}{4 \sqrt{\varepsilon}} y-\frac{1}{8 \varepsilon \sqrt{\varepsilon}} y^{2}, & y<\varepsilon, \\ \sqrt{x}, & y \geq \varepsilon .\end{cases}
$$

For any $\varepsilon>0, g_{\varepsilon}(y) \in C^{2}(\mathbb{R})$, and $\lim _{\varepsilon \longrightarrow 0} g_{\varepsilon}(y)=\sqrt{y}$ for any $x \geq 0$. Applying (55) to $g_{\varepsilon}\left(R_{t}^{2}\right)$, we obtain 


$$
g_{\varepsilon}\left(R_{t}^{2}\right)=\frac{3}{8} \sqrt{\varepsilon}+\sum_{i=1}^{d} \mathrm{I}(i, \varepsilon)+\mathrm{II}(\varepsilon)+\mathrm{III}(\varepsilon)
$$

where

$$
\begin{aligned}
& \mathrm{I}(i, \varepsilon):=\int_{0}^{t}\left[1_{\left\{R_{s}^{2}<\varepsilon\right\}} \frac{1}{2 \sqrt{\varepsilon}}\left(3-\frac{R_{s}^{2}}{\varepsilon}\right)+1_{\left\{R_{s}^{2} \geq \varepsilon\right\}} \frac{1}{R_{s}}\right] B_{s}^{i} \mathrm{~d} B_{s}^{i}, \\
& \mathrm{II}(\varepsilon):=\mathrm{HK}(d-1) \int_{0}^{t} 1_{\left\{R_{s}^{2} \geq \varepsilon\right\}} \frac{1}{R_{s}} B^{2 \mathrm{HK}-1} \mathrm{~d} s, \\
& \mathrm{III}(\varepsilon):=\mathrm{HK} \int_{0}^{t} 1_{\left\{R_{s}^{2}<\varepsilon\right\}} \frac{1}{2 \sqrt{\varepsilon}}\left[3 d-(d+2) \frac{R_{s}^{2}}{\varepsilon}\right] B^{2 \mathrm{HK}-1} \mathrm{~d} .
\end{aligned}
$$

Together with $\int_{0}^{t}\left(s^{2 \mathrm{HK}-1} / R_{s}\right)<\infty$ a.s. and the bounded convergence theorem, one can obtain

$$
\lim _{\varepsilon \longrightarrow 0} \mathrm{II}(\varepsilon)=(d-1) \mathrm{HK} \int_{0}^{t} \frac{1}{R_{s}} s^{2 \mathrm{HK}-1} \mathrm{~d} s .
$$

For the third term, by the substituting $u=\left(\rho / s^{H, K}\right)$ and Fubini's theorem, we can obtain

$$
\begin{aligned}
0 & \leq E(\mathrm{III}(\varepsilon)) \leq \frac{3 \mathrm{HKd}}{2 \sqrt{\varepsilon}} \int_{0}^{t} P\left\{R_{s}^{2}<\varepsilon\right\} s^{2 \mathrm{HK}-1} \mathrm{~d} s \\
& \leq \frac{3 \mathrm{HKd}}{2 \sqrt{\varepsilon}} \int_{0}^{t} P\left\{\left(B_{s}^{1}\right)^{2}+\left(B_{s}^{2}\right)^{2}<\varepsilon\right\} s^{2 \mathrm{HK}-1} \mathrm{~d} s \\
& =\frac{3 \mathrm{HKd}}{2 \sqrt{\varepsilon}} \int_{0}^{t}\left[\int_{0}^{2 \pi} \int_{0}^{\sqrt{\varepsilon}} \frac{\rho}{2 \pi s^{2 \mathrm{HK}}} e^{-\left(\rho^{2} / 2 B_{s}^{2 H K}\right)} \mathrm{d} \rho \mathrm{d} \theta\right] s^{2 \mathrm{HK}-1} \mathrm{~d} s \\
& \leq \frac{3 d}{2 \sqrt{\varepsilon}} \int_{0}^{\sqrt{\varepsilon}}\left(\int_{\frac{\rho}{t^{H K}}}^{\infty} \frac{1}{u} e^{-\left(u^{2} / 2\right)} \mathrm{d} u\right) \rho \mathrm{d} \rho,
\end{aligned}
$$

that is,

$$
\lim _{\varepsilon \longrightarrow 0} E(|\operatorname{III}(\varepsilon)|)=0 .
$$

Finally, we show that

$$
\lim _{\varepsilon \longrightarrow 0} \mathrm{I}(i, \varepsilon)=\int_{0}^{t} \frac{B_{s}^{i}}{R_{s}} \mathrm{~d} B_{s}^{i}
$$

in $L^{(1 / \mathrm{HK})}(\Omega)$. We have

$$
\begin{aligned}
A(\varepsilon) & :=E\left[\int_{0}^{T}\left|1-\frac{R_{s}}{2 \sqrt{\varepsilon}}\left(3-\frac{R_{s}^{2}}{\varepsilon}\right)\right|^{(1 / \mathrm{HK})}\left|\frac{B_{s}^{i}}{R_{s}}\right|^{(1 / \mathrm{HK})} 1_{\left\{R_{s}^{2}<\varepsilon\right\}} \mathrm{d} s\right] \\
& \leq \int_{0}^{T} P\left\{R_{s}^{2}<\varepsilon\right\} \mathrm{d} s \leq \int_{0}^{T} P\left\{\left(B_{s}^{1}\right)^{2}+\left(B_{s}^{2}\right)^{2}<\varepsilon\right\} \mathrm{d} s \\
& =\int_{0}^{T}\left[\int_{0}^{2 \pi} \int_{0}^{\sqrt{\varepsilon}} \rho \frac{1}{2 \pi B^{2 \mathrm{HK}}} e^{-\left(\rho^{2} / 2 B^{2 \mathrm{HK}}\right)} \mathrm{d} \rho \mathrm{d} \theta\right] \mathrm{d} s \longrightarrow 0,
\end{aligned}
$$

as $\varepsilon \longrightarrow 0$. On the other hand, one can obtain

$$
\begin{aligned}
B(\varepsilon) & :=E\left[\int_{0}^{T} \int_{0}^{T}\left|D_{B}^{i}\left(\left(\frac{B_{r}^{i}}{R_{r}}-\frac{B_{r}^{i}}{2 \sqrt{\varepsilon}}\left(3-\frac{R_{r}^{2}}{\varepsilon}\right)\right) 1_{\left\{R_{r}^{2}<\varepsilon\right\}}\right)\right|^{(1 / \mathrm{HK})} \mathrm{d} s \mathrm{~d} r\right] \\
& =\int_{0}^{T} r E\left[\left|\left(\frac{1}{R_{r}}-\frac{\left(B_{r}^{i}\right)^{2}}{R_{r}^{3}}\right)-\frac{3}{2 \sqrt{\varepsilon}}+\frac{R_{r}^{2}+2\left(B_{r}^{i}\right)^{2}}{2 \varepsilon \sqrt{\varepsilon}}\right|^{(1 / \mathrm{HK})} 1_{\left\{R_{r}^{2}<\varepsilon\right\}}\right] \mathrm{d} r \\
& \leq \int_{0}^{T} r E\left[\left|\frac{1}{R_{r}}+\frac{3}{2 \sqrt{\varepsilon}}+\frac{3 R_{r}^{2}}{2 \varepsilon \sqrt{\varepsilon}}\right|^{(1 / \mathrm{HK})} 1_{\left\{R_{r}^{2}<\varepsilon\right\}}\right] \mathrm{d} r \\
& \leq 4^{\frac{1}{\mathrm{HK}}} \int_{0}^{T} E\left[\left|R_{1}\right|^{-(1 / \mathrm{HK})} 1_{\left\{R_{1}^{2}<\left(\varepsilon / r^{2 \mathrm{HK}}\right)\right\}}\right] \mathrm{d} r .
\end{aligned}
$$

The distribution of $\left(B_{1}^{1}, \ldots, B_{1}^{d}\right)$ in spherical coordinates yields

$$
\int_{0}^{T} E\left[\left|R_{1}\right|^{-(1 / \mathrm{HK})} 1_{\left\{R_{1}<\left(\sqrt{\varepsilon} / r^{\mathrm{HK}}\right)\right\}}\right] d r \leq C_{d, H, K} \int_{0}^{T} \int_{0}^{\left(\sqrt{\varepsilon} / r^{\mathrm{HK}}\right)} e^{-\left(u^{2} / 2 l\right)} u^{d-1-(1 / \mathrm{HK})} \mathrm{d} u \mathrm{~d} r,
$$


where $C_{d, H, K}>0$ is a constant which depends on $H, K$, and $d$.

For any $r \in[0, T]$,

$$
\begin{aligned}
& \int_{0}^{\left(\sqrt{\varepsilon} / r^{\mathrm{HK}}\right)} e^{-\left(u^{2} / 2 l\right)} u^{d-1-(1 / \mathrm{HK})} \mathrm{d} u \longrightarrow 0, \quad \varepsilon \longrightarrow 0, \\
& \int_{0}^{\left(\sqrt{\varepsilon} / r^{\mathrm{HK}}\right)} e^{-\left(u^{2} / 2 l\right)} u^{d-1-(1 / \mathrm{HK})} \mathrm{d} u \leq \int_{0}^{\infty} e^{-\left(u^{2} / 2 l\right)} u^{d-1-(1 / \mathrm{HK})} \mathrm{d} u<\infty .
\end{aligned}
$$

We have

$$
\lim _{\varepsilon \longrightarrow 0} \int_{0}^{T} E\left[\left|R_{1}\right|^{-(1 / \mathrm{HK})} 1_{\left\{R_{1}<\left(\sqrt{\varepsilon} / r^{\mathrm{HK}}\right)\right\}}\right] \mathrm{d} r=0,
$$

by the bounded convergence theorem, that is,

$$
\lim _{\varepsilon \longrightarrow 0} B(\varepsilon)=0 .
$$

This proves the desired convergence (68), and the proposition follows.

Proposition 7. Stochastic process $X$ which is given by (54) is HK-self-similar.
Proof. Set $a>0$. Together with the HK-self-similarity property of the bifractional Brownian motion and (56), we can obtain

$$
\begin{aligned}
X_{\mathrm{at}} & =R_{\mathrm{at}}-\mathrm{HK}(d-1) \int_{0}^{a t} \frac{B_{s}^{2 \mathrm{HK}-1}}{R_{s}} \mathrm{~d} s \\
& =R_{\mathrm{at}}-\mathrm{HK}(d-1) a^{2 \mathrm{HK}} \int_{0}^{t} \frac{u^{2 \mathrm{HK}-1}}{R_{\mathrm{au}}} \mathrm{d} u \\
& \stackrel{d}{=} a^{\mathrm{HK}} R_{t}-\mathrm{HK}(d-1) a^{H} \int_{0}^{t} \frac{u^{2 \mathrm{HK}-1}}{R_{u}} \mathrm{~d} u=a^{\mathrm{HK}} X_{t} .
\end{aligned}
$$

For $h \in \mathscr{H}^{\otimes n}$, we denote

$$
I_{i_{1}, \ldots, i_{k}}(h)=\int_{0<s_{1}, \ldots, s_{k}<t} h\left(s_{1}, \ldots, s_{k}\right) \mathrm{d} B_{s_{1}}^{i_{1}}, \ldots, \mathrm{d} B_{s_{n}}^{i_{n}}, \quad 1 \leq i_{1}, \ldots, i_{k} \leq d .
$$

Theorem 3. Suppose $f_{j}: \mathbb{R}^{d} \longrightarrow \mathbb{R}, j=1,2, \ldots, d$ are with polynomial growth and smooth functions. Then, the stochastic process $Z_{t}=\sum_{j=1}^{d} \int_{0}^{t} f_{j}\left(B_{s}\right) d B_{s}^{j}$ has the following chaos expansion:

$$
Z_{t}=\sum_{j=1}^{d} \sum_{k=1}^{\infty} \sum_{1 \leq i_{1}, \ldots, i_{i} \leq d} I_{i_{1}, \ldots, i_{k}, \mathrm{ji}}\left(g_{i_{1}, \ldots, i_{n}}^{j}\left(B_{1}, \ldots, B_{k+1}\right)\right),
$$

where

$$
\begin{aligned}
& g_{i_{1}, \ldots, i_{k}}^{j}\left(B_{1}, \ldots, B_{k+1}\right)=\frac{(-1)^{n}\left(B_{1} \vee \cdots \vee B_{i+1}\right)^{-\mathrm{kHK}}}{(2 \pi)^{(d / 2)}} \\
& \quad \times \int_{\mathbb{R}^{d}}\left[\frac{\partial^{k}}{\partial y_{i_{1}}, \ldots, \partial y_{i_{k}}} e^{-\left(|y|^{2} / 2\right)}\right] f_{i}\left(y\left(B_{1} \vee \cdots \vee B_{k+1}\right)^{\mathrm{HK}}\right) \mathrm{d} y .
\end{aligned}
$$

Proof. For each $(j=1,2, \ldots, d)$, using Stroock's formula, we can obtain

$$
f_{j}\left(B_{s}\right)=\sum_{k=0}^{\infty} \sum_{1 \leq i_{1}, \ldots, i_{k} \leq d} \frac{1}{k !} I_{i_{1}, \ldots, i_{n}}\left(f_{i_{1}, \ldots, i_{n}}^{i}(s) 1_{[0, s]}^{\otimes n}\right)
$$

$$
\begin{aligned}
f_{i_{1}, \ldots, i_{k}}^{j}(s) & =E\left(D^{i_{1}}, \ldots, D^{i_{n}}\left(f_{j}\left(B_{s}\right)\right)\right) \\
& =E\left(\frac{\partial^{n} f_{j}}{\partial z_{i_{1}}, \ldots, \partial z_{i_{k}}}\left(B_{s}\right)\right) \\
& =\frac{1}{\left(2 \pi \mathrm{lB} \mathrm{B}^{2 \mathrm{HK}}\right)^{(d / 2)}} \int_{\mathbb{R}^{d}} \frac{\partial^{n} f_{i}}{\partial z_{i_{1}}, \ldots, \partial z_{i_{n}}}(z) e^{-\left(|z|^{2} / 2 s^{2 \mathrm{HK}}\right)} d z \\
& =\frac{(\sqrt{l})^{-n} B^{-\mathrm{nH}}}{(2 \pi)^{(d / 2)}} \int_{\mathbb{R}^{d}} \frac{\partial^{k} f_{i}}{\partial z_{i_{1}}, \ldots, \partial z_{i_{n}}}\left(y B^{H, K}\right) e^{-\left(|y|^{2} / 2\right)} \mathrm{d} y \\
& =\frac{(-1)^{n} B^{-\mathrm{nH}}}{(2 \pi)^{(d / 2)}} \int_{\mathbb{R}^{d}} f_{j}\left(y B^{H, K}\right)\left[\frac{\partial^{n}}{\partial z_{i_{1}}, \ldots, \partial z_{i_{n}}} e^{-\left(|y|^{2} / 2\right)}\right] \mathrm{d} y .
\end{aligned}
$$

So,

$$
\begin{aligned}
Z_{t} & =\sum_{j=1}^{d} \int_{0}^{t} f_{j}\left(B_{s}\right) \mathrm{d} B_{s}^{j}, \\
& \left.=\sum_{i=1}^{d} \sum_{k=0}^{\infty} \sum_{1 \leq i_{1}, \ldots, i_{n} \leq d} I_{i_{1}, \ldots, i_{k}, i}\left(\operatorname{symm}\left(f_{i_{1}, \ldots, i_{k}}^{i}(s)\right) 1_{[0, s]}^{\otimes n}\right]_{[0, t]}(s)\right) \\
& =\sum_{i=1}^{d} \sum_{k=0}^{\infty} \sum_{1 \leq i_{1}, \ldots, i_{n} \leq d} I_{i_{1}, \ldots, i_{n}, i}\left(f_{i_{1}, \ldots, i_{k}}^{i}\left(B_{1} \vee \cdots \vee B_{k+1}\right) \prod_{j=1}^{k+1} 1_{[0, t]}\left(B_{j}\right)\right) .
\end{aligned}
$$

where
This completes the proof. 
Let $f_{j}(x)=\left(x_{j} / \sqrt{x_{1}^{2}+\cdots+x_{d}^{2}}\right)$; then, $f_{j}(t x)=f_{j}(x)$. So, for such $f_{j}$, one can obtain

$$
g_{i_{1}, \ldots, i_{k}}^{j}\left(B_{1}, \ldots, B_{k+1}\right)=\frac{(-1)^{k}(\sqrt{l})^{-k}\left(B_{1} \vee \cdots \vee B_{k+1}\right)^{-\mathrm{kHK}}}{(2 \pi)^{(d / 2)}} \times \int_{\mathbb{R}^{d}}\left[\frac{\partial^{k}}{\partial y_{i_{1}} \cdots \partial y_{i_{n}}} e^{-\left(|y|^{2} / 2\right)}\right] f_{j}(y) \mathrm{d} y .
$$

Then, $\left(B_{t}^{j} / R_{t}\right)$ can be denoted by

$$
\frac{B_{t}^{j}}{R_{t}}=\sum_{k=0}^{\infty} \sum_{1 \leq i_{1}, \ldots, i_{n} \leq d} \frac{(-1)^{k}(t)^{-\mathrm{kHK}}}{(2 \pi)^{(d / 2)}} \times \int_{\mathbb{R}^{d}}\left[\frac{\partial^{n}}{\partial y_{i_{1}}, \ldots, \partial y_{i_{n}}} e^{-\left(|y|^{2} / 2\right)}\right] f_{j}(y) \mathrm{d} y \int_{\left\{0<s_{1}<\cdots<s_{k}<t\right\}} \mathrm{d} B_{s_{1}}^{i_{1}}, \ldots, \mathrm{d} B_{s_{k}}^{i_{n}},
$$

and the chaos expansion of $\int_{0}^{t}\left(B_{B}^{i} / R_{s}\right) \mathrm{d} B_{s}^{i}$ is

$$
\int_{0}^{t} \frac{B_{s}^{i}}{R_{s}} \mathrm{~d} B_{s}^{i}=\sum_{n=1}^{\infty} \sum_{1 \leq j_{1}, \ldots, j_{k} \leq d} \frac{(-1)^{k}}{(2 \pi)^{(d / 2)}} \int_{\mathbb{R}^{d}}\left[\frac{\partial^{k}}{\partial y_{j_{1}}, \ldots, \partial y_{j_{n}}} e^{-\frac{|y|^{2}}{2}}\right] f_{i}(y) \mathrm{d} y \times \int_{\left\{0<s_{1}, \ldots, s_{n}, s_{k+1}<t\right\}}\left(s_{1} \vee \cdots \vee s_{k+1}\right)^{-\mathrm{kHK}} \mathrm{d} B_{s_{1}}^{j_{1}}, \ldots, \mathrm{d} B_{s_{k}}^{j_{n}} \mathrm{~d} B_{s_{k+1}}^{i}
$$

The theorem is proved.

$$
\rho_{k}=E\left[\sum_{i=1}^{d} \int_{0}^{1} \frac{B_{s}^{i}}{\left|B_{s}\right|} \mathrm{d} B_{s}^{i} \sum_{i=1}^{d} \int_{k}^{k+1} \frac{B_{s}^{i}}{\left|B_{s}\right|} \mathrm{d} B_{s}^{i}\right] .
$$

Theorem 4. The stochastic process $X$ is short-range dependent.

For every $K \geq 1$, by the formula, we can decompose $\rho_{k}$ as

Proof. Let

$$
\begin{aligned}
\rho_{k} & =\sum_{i, j=1}^{d} \int_{0}^{1} \int_{k}^{k+1} E\left(\frac{B_{s}^{i} B_{t}^{j}}{\left|B_{s} \| B_{t}\right|}\right) \phi(t, s) \mathrm{d} s \mathrm{~d} t+\sum_{i, j=1}^{d} \int_{0}^{1} \int_{k}^{k+1} \int_{0}^{t} \int_{0}^{B} \phi(s, u) \phi(t, v) E\left(D_{v}^{j}\left(\frac{B_{s}^{i}}{\left|B_{s}\right|}\right) D_{u}^{i}\left(\frac{B_{t}^{j}}{\left|B_{t}\right|}\right)\right) \mathrm{d} u \mathrm{~d} v \mathrm{~d} s \mathrm{~d} t \\
& \equiv \rho_{k, 1}+\rho_{k, 2} .
\end{aligned}
$$

For $\rho_{k, 1}$, one can use the decomposition

$$
B_{t} \stackrel{d}{=} \frac{\theta_{H}(s, t)}{\theta_{H}(s, s)} B_{s}+\beta_{s, t} \mathcal{N}
$$

where

$$
\beta_{s, t}^{2}=\frac{\rho_{s, t}^{2}}{s^{2 \mathrm{HK}}}
$$

where $\mathcal{N}$ is independent of $B_{s}$ and is denoted as ad-dimensional standard normal random variable. By Lemma 13 in [11],

$$
\begin{aligned}
E\left(\frac{\left\langle B_{s}, B_{t}\right\rangle}{\left|B_{s} \| B_{t}\right|}\right) & =E\left(\frac{\left\langle B_{s}, \lambda_{s, t} B_{s}+\mathcal{N}\right\rangle}{\left|B_{s} \| \lambda_{s, t} B_{s}+\mathcal{N}\right|}\right) \\
& =E\left(\frac{\left\langle B_{1}, s^{H, K} \lambda_{s, t} B_{1}+\mathcal{N}\right\rangle}{\left|B_{s} \| s^{H, K} \lambda_{s, t} B_{1}+\mathcal{N}\right|}\right) \\
& =s^{H, K} \lambda_{s, t} E\left(\frac{\left|B_{1}\right|^{2}|\mathcal{N}|^{2}-\left\langle B_{1}, \mathcal{N}\right\rangle^{2}}{\left|B_{s} \| \mathcal{N}\right|^{3}}\right)+O\left(t^{-\mathrm{HK}}\right) .
\end{aligned}
$$


Thus,

$$
E\left(\frac{\left\langle B_{s}, B_{t}\right\rangle}{\left|B_{s} \| B_{t}\right|}\right)=s^{\mathrm{HK}} t^{-\mathrm{HK}},
$$

which implies that the term $\rho_{k, 1}$ behaves as $k^{H-3}$.

For $\rho_{k, 2}$, one has

$$
\begin{aligned}
\rho_{k, 2} & =(\mathrm{HK})^{2} \sum_{i, j=1}^{d} \int_{0}^{1} \int_{k}^{k+1} E\left(\left(\frac{\delta_{i j}}{\left|B_{s}\right|}-\frac{B_{s}^{i} B_{s}^{j}}{\left|B_{s}^{H, K}\right|^{3}}\right)\left(\frac{\delta_{i j}}{\left|B_{t}\right|}-\frac{B_{t}^{i} B_{t}^{j}}{\left|B_{t}\right|^{3}}\right)\right) \psi_{1}(s, t) \psi_{2}(s, t) \mathrm{d} s \mathrm{~d} t \\
& =(\mathrm{HK})^{2} \int_{0}^{1} \int_{k}^{k+1} E\left(\frac{d}{\left|B_{s}\right|\left|B_{t}\right|}-\frac{\left|B_{s}\right|^{2}}{\left|B_{s}\right|^{3}\left|B_{t}\right|}-\frac{\left|B_{t}\right|^{2}}{\left|B_{s}\right|\left|B_{t}\right|^{3}}+\frac{\left\langle B_{s}, B_{t}\right\rangle^{2}}{\left|B_{s}\right|^{3}\left|B_{t}\right|^{3}}\right) \psi_{1}(s, t) \psi_{2}(s, t) \mathrm{d} s \mathrm{~d} t \\
& =(\mathrm{HK})^{2} \int_{0}^{1} \int_{n}^{n+1} E\left(\frac{\left\langle B_{s}, B_{t}\right\rangle^{2}}{\left|B_{s}\right|^{3}\left|B_{t}\right|^{3}}+\frac{d-2}{\left|B_{s}\right|\left|B_{t}\right|}\right) \psi_{1}(s, t) \psi_{2}(s, t) \mathrm{d} s \mathrm{~d} t .
\end{aligned}
$$

Since

$$
E\left(\frac{\left\langle B_{s}, B_{t}\right\rangle^{2}}{\left|B_{s}\right|^{3}\left|B_{t}\right|^{3}}+\frac{d-2}{\left|B_{s} \| B_{t}\right|}\right),
$$

behaves as $M t^{-\mathrm{HK}}$ as $t \longrightarrow \infty$, where

$$
M=E\left(\frac{\left\langle B_{1}, \mathcal{N}\right\rangle^{2}}{\left|B_{1}\right|^{3}|\mathcal{N}|^{3}}+\frac{d-2}{\left|B_{1} \| \mathcal{N}\right|}\right)>0 .
$$

We see that the term $\rho_{k, 2}$ also behaves as $k^{\mathrm{HK}-3}$, and the theorem follows.

\section{The Local Times of $\int_{0}^{t} \operatorname{sign}\left(B_{s}^{H, K}\right) \mathbf{d} B_{s}^{H, K}$}

Now, we consider the local times of the stochastic process $X=\left\{X_{t}, \quad t \geq 0\right\}$ defined by

$$
X_{t}=\int_{0}^{t} \operatorname{sign}\left(B_{s}^{H, K}\right) \mathrm{d} B_{s}^{H, K} .
$$

Lemma 2. Let $((1 / 2)<H K<1)$. Then, for all $t \geq 0$, we have

$$
\operatorname{sign}\left(B_{t}^{H, K}\right) D_{t} X(t) \geq 0 \text {, a.s. }
$$

Proof. Using the Itô formula, one can obtain

$$
\begin{aligned}
X(t)^{2} & =2 \int_{0}^{t} X_{s} \operatorname{sign}\left(B_{s}^{H, K}\right) \mathrm{d} B_{s}^{H, K}+2 \int_{0}^{t} \operatorname{sign}\left(B_{s}^{H, K}\right) D_{s} X_{s} \mathrm{~d} s \\
\sigma(t) & \equiv E(X(t))^{2}=2 E \int_{0}^{t} \operatorname{sign}\left(B_{s}^{H, K}\right) D_{s} X_{s} \mathrm{~d} s \\
& =\int_{0}^{t} \int_{0}^{s} E\left[\operatorname{sign}\left(B_{s}^{H, K}\right) \operatorname{sign}\left(B_{s}^{H, K}\right)\right] \phi(s, u) \mathrm{d} u \mathrm{~d} s
\end{aligned}
$$

and note that the function $t \longrightarrow \sigma(t)$ is increasing since

$$
E\left[\operatorname{sign}\left(B_{s}^{H, K}\right) \operatorname{sign}\left(B_{u}^{H, K}\right)\right]=\sum_{m=1}^{\infty} \frac{\left.4(2 m) !\left(\left(1 / 2^{K}\right)\left[\left(t^{2 H}+s^{2 H}\right)^{K}-|t-s|^{2 \mathrm{HK}}\right]\right)\right)^{2 m+1}}{(2 m+1)^{2} 2 \pi\left(k ! 2^{k}\right)^{2}(\mathrm{su})^{2 m+1}} .
$$

So,

$$
E \int_{t_{0}}^{t} \operatorname{sign}\left(B_{s}^{H, K}\right) D_{s} X_{s} \mathrm{~d} s \geq 0
$$

for all $t \geq t_{0} \geq 0$. Now, let us prove $\left(\operatorname{sign}\left(B_{t}^{H, K}\right) D_{s} X(t) \geq 0\right)$, a.s. We only need to show that

$$
\int_{t_{0}}^{t} \operatorname{sign}\left(B_{s}^{H, K}\right) D_{s} X_{s} \mathrm{~d} s, \quad 0 \leq t \leq T
$$

is nondecreasing. Let

$$
\begin{aligned}
V_{t_{0}}(t) & =\int_{t_{t_{0}}}^{t} \operatorname{sign}\left(B_{s}^{H, K}\right) D_{s} X_{s} \mathrm{~d} s, \quad 0 \leq t_{0} \leq t, \\
\Phi(x) & =1_{x<0}, \\
\Phi_{\varepsilon} & \in C^{2}(R), \\
I & =\Phi\left(V_{t_{0}}(t)\right), \\
I_{\varepsilon} & =\Phi_{\varepsilon}\left(V_{t_{0}}(t)\right),
\end{aligned}
$$

where $\Phi_{\varepsilon}(x)=0$ for $x>\varepsilon$ and $\Phi_{\varepsilon}(x)=1$ for $x<\varepsilon$. Thus, one can obtain 


$$
\begin{array}{r}
\mathrm{ID}_{s} I_{\varepsilon}=0, \\
I \cdot V_{t_{0}}(t) \leq 0 .
\end{array}
$$

So, for all $t \geq t_{0} \geq 0$, one can obtain

$$
\begin{aligned}
E\left[\operatorname{IV}_{t_{0}}(t)\right] & =E \int_{t_{0}}^{t} I \operatorname{sign}\left(B_{s}^{H, K}\right) D_{s} X_{s} \mathrm{~d} s \\
& =\lim _{\varepsilon \longrightarrow 0} E \int_{t_{0}}^{t} I I_{\varepsilon} \operatorname{sign}\left(B_{s}^{H, K}\right) D_{s} X_{s} \mathrm{~d} s \\
& \geq E \int_{t_{0}}^{t} I \operatorname{sign}\left(B_{s}^{H, K}\right) D_{s} X_{s} \mathrm{~d} s \geq 0,
\end{aligned}
$$

which implies that $I=0$ and $V_{t_{0}}(t) \geq 0$. Therefore,

$$
\int_{t_{0}}^{t} \operatorname{sign}\left(B_{s}^{H, K}\right) D_{s} X_{s} \mathrm{~d} s, \quad 0 \leq t \leq T
$$

is nondecreasing. This completes the proof.

Theorem 5. Let the stochastic process $X=\left\{X_{t}, t \geq 0\right\}$ be defined by

$$
X_{t}=\int_{0}^{t} \operatorname{sign}\left(B_{s}^{H, K}\right) \mathrm{d} B_{s}^{H, K},
$$

and let $\Phi: R^{+} \longrightarrow R$ be a convex function with polynomial growth. Then, there is a continuous increasing process $A^{\Phi}$ which satisfies

$$
\Phi\left(X_{t}\right)=\Phi(0)+\int_{0}^{t} D^{-}\left(X_{s}\right) \operatorname{sign}\left(B_{s}^{H, K}\right) \mathrm{d} B_{s}^{H, K}+\frac{1}{2} A_{t}^{\Phi}
$$

where $D^{-} \Phi$ denotes the left-hand derivative of $\Phi$.

Proof. If $\Phi \in C^{2}$, then this is the Ito formula, and

$$
A_{t}^{\Phi}=\int_{0}^{t} \Phi^{\prime \prime}\left(X_{s}\right) \operatorname{sign}\left(B_{s}^{H, K}\right) D_{s} X_{s} \mathrm{~d} s
$$

together with Lemma 2, implies that the stochastic process $A^{\Phi}$ is increasing.

Now, let $\Phi \notin C^{2}$. For $x \in R$ and $\varepsilon>0$, one sets

$$
\begin{aligned}
& p_{\varepsilon}(x)=\frac{1}{\sqrt{2} \pi \varepsilon} e^{-(1 / 2 \varepsilon) x^{2}}, \\
& \Phi_{\varepsilon}(x)=\int_{R} p_{\varepsilon}(x-y) \Phi(y) \mathrm{d} y .
\end{aligned}
$$

It is easy to see that $\Phi_{\varepsilon} \in C^{2}$ and has polynomial growth. So, for all $\varepsilon>0$, there exists a continuous increasing process $A^{\Phi_{\varepsilon}}$ such that

$$
\begin{aligned}
\Phi_{\varepsilon}\left(X_{t}\right) & =\Phi_{\varepsilon}(0)+\int_{0}^{t} \Phi_{\varepsilon}^{\prime}\left(X_{s}\right) \operatorname{sign}\left(B_{s}^{H, K}\right) \mathrm{d} B_{s}^{H, K}+\frac{1}{2} A_{t}^{\Phi_{\varepsilon}}, \\
A_{t}^{\Phi_{\varepsilon}} & =\int_{0}^{t} \Phi_{\varepsilon}^{\prime \prime}\left(X_{s}\right) \operatorname{sign}\left(B_{s}^{H, K}\right) D_{s} X_{s} \mathrm{~d} s \\
& =\int_{R} \Phi_{\varepsilon}^{\prime \prime}(x)\left(\int_{0}^{t} \delta\left(X_{s}-x\right)\right)\left(\operatorname{sign}\left(B_{s}^{H, K}\right)\right) D_{s} X_{s} \mathrm{~d} s \mathrm{~d} x .
\end{aligned}
$$

Note that

$$
\lim _{\varepsilon \downarrow 0} \Phi_{\varepsilon}(x)=\Phi(x), \lim _{\varepsilon \downarrow 0} \Phi_{\varepsilon}^{\prime}(x)=D^{-} \Phi(x),
$$

and one can obtain as $\varepsilon \longrightarrow 0$

$$
\int_{0}^{t} \Phi_{\varepsilon}^{\prime}\left(X_{s}\right) \operatorname{sign}\left(B_{s}^{H, K}\right) \mathrm{d} B_{s}^{H, K} \longrightarrow \int_{0}^{t} D^{-}\left(X_{s}\right) \operatorname{sign}\left(B_{s}^{H, K}\right) \mathrm{d} B_{s}^{H, K},
$$

in probability. So, $A^{\Phi_{\varepsilon}}$ converges to a stochastic process $A^{\Phi}$ which, as a limit of increasing stochastic processes, is itself an increasing stochastic process and

$$
\Phi\left(X_{t}\right)=\Phi(0)+\int_{0}^{t} D^{-}\left(X_{s}\right) \operatorname{sign}\left(B_{s}^{H, K}\right) \mathrm{d} B_{s}^{H, K}+\frac{1}{2} A_{t}^{\Phi},
$$

where $A^{\Phi}$ can be chosen to be a.s. continuous. The proof is completed.

Corollary 1. For the process $X_{t}=\int_{0}^{t} \operatorname{sign}\left(B_{s}^{H, K}\right) d B_{s}^{H, K}$ and all $x \in R$, there exists a local time $\mathscr{L}^{x}(X)$ such that

$$
\left|X_{t}-x\right|=|x|+\int_{0}^{t} \operatorname{sign}\left(X_{s}-x\right) \mathrm{d} X_{s}+\mathscr{L}_{t}^{x}(X) .
$$

Proof. Note that the left derivative of the function $\Phi(y)=$ $(y-x)^{+}$is equal to $1_{x, \infty}(y)$. By Theorem 5 , one can obtain

$$
\left(X_{t}-x\right)^{+}=(-x) \vee 0+\int_{0}^{t} 1_{X_{s}>x} \operatorname{sign}\left(B_{s}^{H, K}\right) \mathrm{d} B_{s}^{H, K}+\frac{1}{2} A^{+},
$$

where $A^{+}$is a continuous increasing stochastic process. Similarly, there exists a continuous increasing stochastic process $A^{-}$which satisfies

$$
\left(X_{t}-x\right)^{-}=(x) \vee 0+\int_{0}^{t} 1_{X_{s}>x} \operatorname{sign}\left(B_{s}^{H, K}\right) \mathrm{d} B_{s}^{H, K}+\frac{1}{2} A^{-} .
$$

Therefore, one can obtain

$$
X_{t}=\int_{0}^{t} \operatorname{sign}\left(B_{s}^{H, K}\right) \mathrm{d} B_{s}^{H, K}+\frac{1}{2}\left(A^{+}+A^{-}\right),
$$

which implies that $A^{+}=A^{-}$a.s. and we set $\mathscr{L}_{x}^{x}(X)=A_{t}^{+}$. This completes the proof.

Combining this corollary with Es-Sebaiy and Tudor [3], we can obtain the following results. 
Corollary 2. Suppose that $\mathscr{L}^{x}(X)$ is the local time of the process $X$ and $\mathscr{L}^{x}\left(B^{H, K}\right)$ is the weighted local time of the bifractional Brownian motion $B^{H, K}$ defined by

$$
\mathscr{L}_{t}^{x}\left(B^{H, K}\right)=2 \mathrm{HK} \int_{0}^{t} \delta\left(B^{H, K}-x\right) s^{2 \mathrm{HK}-1} \mathrm{~d} s .
$$

$$
\begin{aligned}
\mathscr{L}_{t}^{x}(X)-\mathscr{L}_{t}^{x}\left(B^{H, K}\right)= & \left|X_{t}-x\right|-\left|B^{H, K}-x\right| \\
& +2 \int_{0}^{t} 1_{\left\{X_{s} \leq x\right\}} \operatorname{sign}\left(B_{s}^{H, K}-x\right) \mathrm{d} B_{s}^{H, K} .
\end{aligned}
$$

Then, we have

$$
\begin{aligned}
\left|X_{t}-x\right|-\left|B^{H, K}-x\right|= & \mathscr{L}_{t}^{x}(X)-\mathscr{L}_{t}^{x}\left(B^{H, K}\right) \\
& +\int_{0}^{t} \operatorname{sign}\left(X_{s}-x\right) \operatorname{sign}\left(B_{s}^{H, K}-x\right) \mathrm{d} B_{s}^{H, K}-\int_{0}^{t} \operatorname{sign}\left(B_{s}^{H, K}-x\right) \mathrm{d} B_{s}^{H, K} \\
= & \mathscr{L}_{t}^{x}(X)-\mathscr{L}_{t}^{x}\left(B^{H, K}\right)+\int_{0}^{t}\left[\operatorname{sign}\left(X_{s}-x\right)-1\right] \operatorname{sign}\left(B_{s}^{H, K}-x\right) \mathrm{d} B_{s}^{H, K} \\
= & \mathscr{L}_{t}^{x}(X)-\mathscr{L}_{t}^{x}\left(B^{H, K}\right)-2 \int_{0}^{t} 1_{\left\{X_{s} \leq x\right\}} \operatorname{sign}\left(B_{s}^{H, K}-x\right) \mathrm{d} B_{s}^{H, K},
\end{aligned}
$$

which implies that (117) holds.

Corollary 3. For any $t \geq 0$ and $x \in R$, we have

$$
\mathscr{L}_{t}^{x}(X)=\int_{0}^{t} \delta(X(s)-x) \operatorname{sign}\left(B_{s}^{H, K}\right) D_{s} X_{s} \mathrm{~d} s .
$$

Moreover, let $\Phi: R^{+} \longrightarrow R$ be a convex function with polynomial growth; one can obtain the following Itô-Tanaka formula:

$$
\begin{aligned}
\Phi(X(t))= & \Phi(0)+\int_{0}^{t} D^{-} \Phi\left(X_{s}\right) \operatorname{sign}\left(B_{s}^{H, K}\right) \mathrm{d} B_{s}^{H, K} \\
& +\frac{1}{2} \int_{R} \mathscr{L}_{t}^{x}(X) \mu_{\Phi} \mathrm{d} x,
\end{aligned}
$$

where $D^{-} \Phi$ denotes the left derivative of $\Phi$ and signed measure $\mu_{\Phi}$ which is defined by

$$
\mu_{\Phi}([a, b])=D^{-} \Phi(b)-D^{-} \Phi(a), \quad a<b, a, b \in R .
$$

Finally, one can prove that the local time of the process

$$
\sum_{i=1}^{d} \int_{0}^{t} \frac{B_{s}^{i}}{R_{s}} \mathrm{~d} B_{s}^{i}, \quad d \geq 2
$$

exists by the same method and can obtain the similar results.

\section{Conclusions}

This paper presents theorems and propositions associated with respect to the stochastic process $R_{t}=$ $\sqrt{\left(B_{t}^{1}\right)^{2}+\cdots+\left(B_{t}^{d}\right)^{2}}$, where $B=\left\{\left(B_{t}^{1}, \ldots, B_{t}^{d}\right)\right\}_{t \geq 0}$ is a $d$-dimensional bifractional Brownian motion and $(2 \mathrm{HK} \geq 1)$. Since there is no Lévy's characterization theorem for a general bifractional Brownian motion, to prove whether a stochastic process

$$
X_{t}:=\sum_{i=1}^{a} \int_{0}^{t} \frac{B_{s}^{i}}{R_{s}} \mathrm{~d} B_{s}^{i}, \quad d \geq 1
$$

is a bifractional Brownian motion or not is difficult. Theorems 1 and 4 prove $X_{t}$ is short-range dependent in one-dimensional case and multidimensional case, respectively. Theorem 5 considers the local times of the stochastic process of $X_{t}$ in one-dimensional case. Theorem 2 gives the the following chaos expansion of the stochastic process $Z_{t}=\sum_{i=1}^{d} \int_{0}^{t} f_{i}\left(B_{s}\right) \mathrm{d} B_{s}^{i}$. Moreover, significance results associated with the above theorem are given.

\section{Data Availability}

All the data generated during this study are included within this article.

\section{Conflicts of Interest}

The authors declare that they have no conflicts of interest.

\section{Authors' Contributions}

All the authors contributed equally and significantly in writing this paper. All the authors read and approved the final manuscript.

\section{Acknowledgments}

Xichao Sun acknowledges the National Natural Science Foundation of China (11971101 and 11426036), Natural Science Foundation of Anhui Province (1808085MA02), and Quality Engineering of Anhui Education (2018jyxm0497 and 2020zdxsjg214). Ming Li acknowledges the National Natural Science Foundation of China under grant nos. 61672238, 61272402, and 61070214. 


\section{References}

[1] C. Houdré and J. Villa, "An example of infinite dimensional quasi-helix," in Stochastic Models (Mexico City, 2002), pp. 195-201, American Mathematical Society, 2003American Mathematical Society, Providence, RI, USA, Contemporary Mathematics, 336.

[2] S. Aazizi and K. Es-Sebaiy, "Berry-Ess'een bounds and almost sure CLT for the quadratic variation of the bifractional Brownian motion," Random Operators and Stochastic Equations, vol. 24, pp. 1-13, 2012.

[3] K. Es-Sebaiy and C. A. Tudor, "Multidimensional bifractional Brownian motion: itô and Tanaka formulas," Stochastics and Dynamics, vol. 7, no. 3, pp. 366-388, 2007.

[4] J. Guo, "Stochastic current of bifractional brownian motion," Journal of Applied Mathematics, vol. 2014, Article ID 762484, 10 pages, 2014.

[5] Y. Jiang and Y. Wang, "Self-intersection local times and collision local times of bifractional Brownian motions," Science in China Series A: Mathematics, vol. 52, no. 9, pp. 1905-1919, 2009.

[6] I. Kruk, F. Russo, and C. A. Tudor, "Wiener integrals, Malliavin calculus and covariance measure structure," Journal of Functional Analysis, vol. 249, no. 1, pp. 92-142, 2007.

[7] F. Russo and C. A. Tudor, "On bifractional Brownian motion," Stochastic Processes and Their Applications, vol. 116, no. 5, pp. 830-856, 2006.

[8] C. A. Tudor and Y. Xiao, "Sample path properties of bifractional Brownian motion," Bernoulli, vol. 13, no. 4, pp. 1023-1052, 2007.

[9] L. Yan, B. Gao, and J. Liu, "The Bouleau-Yor identity for a bifractional Brownian motion," Stochastics, vol. 86, no. 3, pp. 382-414, 2014.

[10] X. Yu, X. Sun, and L. Yan, "Solving a stochastic heat equation driven by a bi-fractional noise," Boundary Value Problems, vol. 2016, no. 1, 2016.

[11] Y. Hu and D. Nualart, "Some processes associated with fractional Bessel processes," Journal of Theoretical Probability, vol. 18, no. 2, pp. 377-397, 2005.

[12] X. Peng, W. Su, W. Su, and Z. Zhang, "On a perturbed compound Poisson risk model under a periodic thresholdtype dividend strategy," Journal of Industrial \& Management Optimization, vol. 16, no. 4, pp. 1967-1986, 2020.

[13] J. Pitman and M. Yor, "Bessel processes and infinitely divisible laws," in Lecture Notes in MathematicsSpringer, Berlin, Germany, 1981.

[14] D. Revuz and M. Yor, Continuous Martingales and Brownian Motion, Springer, Berlin, Germany, 3rd edition, 1999.

[15] M. Yor, Some Aspects of Brownian Motion. Part II: Some Recent Martingale Problems, Birkhäuser, Basel, Switzerland, 1997.

[16] E. Alòs, O. Mazet, and D. Nualart, "Stochastic calculus with respect to Gaussian processes," The Annals of Probability, vol. 29, no. 2, pp. 766-801, 2001.

[17] G. Shen, C. Chen, and L. Yan, "Remarks on sub-fractional bessel processes," Acta Mathematica Scientia, vol. 31, no. 5, pp. 1860-1876, 2011.

[18] D. Nualart, Malliavin Calculus and Related Topics, Springer, New York, NY, USA, 2nd edition, 2006.

[19] J. M. E. Guerra and D. Nualart, "The $(1 / H)$ variation of the divergence integral with respect to the fractional Brownian motion for $H>(1 / 2)$ and fractional Bessel processes," Stochastic Processes and Their Applications, vol. 115, pp. 91-115, 2005. 